Revista Signos

2010, 43(72)

49-69

\title{
Productividad del sufijo -ero en la neología del español de Argentina, Chile y Uruguay ${ }^{1}$
}

\author{
Mary Fuentes \\ Paola Cañete \\ Constanza Gerding \\ Gabriela Kotz \\ Alma Pecchi \\ Universidad de Concepción \\ Chile
}

Resumen: En este artículo se presenta el resultado del análisis de las propiedades morfológicas y semánticas del sufijo -ero en creaciones neológicas por sufijación en las variedades del español de Argentina, Chile y Uruguay, como una manera de verificar su vitalidad. Para ello, se estudió las creaciones neológicas que incluyen este sufijo de un corpus constituido por 147 unidades, relevadas de seis periódicos de Argentina, Chile y Uruguay. En todos los casos se consignó la frecuencia de las formas (type frequency) y la frecuencia de los usos (token frequency). Luego se analizó los distintos valores del sufijo -ero, lo que dio por resultado una taxonomía de cuatro categorías diferentes. Finalmente, se comparó las unidades neológicas terminadas en -ero y en -ista adjuntadas a una misma base léxica y se comprobó la coexistencia de ambas formas. El valor del sufijo -ero que corresponde a profesión u oficio presenta la más alta productividad. Las nuevas unidades formadas con -ero corresponden tanto a la categoría de neología denominativa como a la de variación estilística. Los resultados sugieren que la alta productividad del sufijo -ero se debería a que, por estar integrado a la gramática de los hablantes, estos recurren a dicho formante con gran facilidad.

Palabras Clave: Neología, morfología derivativa, type frequency, token frequency, vitalidad lingüística.

Recibido: 10-XI-2008

Aceptado: 27-X-2009
Correspondencia: Mary Fuentes (marfuent@udec.cl). Facultad de Humanidades y Arte, Universidad de Concepción. Casilla 160-C, Correo 3, Concepción, Chile. 


\title{
Productivity of the Spanish suffix '-ero' in neological coinages in Argentinian, Chilean and Uruguayan Spanish
}

\begin{abstract}
This study analyses the morphological and semantic features of the Spanish suffix '-ero' in neologisms resulting from suffixation produced in Argentinean, Chilean and Uruguayan Spanish, as a way of verifying their vitality. For this purpose, neological coinages, including this suffix from a corpus of 147 items, collected from six newspapers from Argentina, Chile and Uruguay, are studied. Both the type frequency and the token frequency were recorded for all the cases. Then the different meanings of the suffix '-ero' were analyzed and classified into four different categories. Finally, the coinages ending in '-ero' and '-ista' attached to the same root were compared and contrasted, and the possibility of their co-occurrence was observed. The most productive value for '-ero' is that which refers to a profession or activity. These new terms fall into the categories of denominative neology and stylistic variation. The results suggest that the high productivity of the suffix '-ero' might be due to the fact that, as it is integrated into the morphosyntactic system of the speakers, they easily resort to it.
\end{abstract}

Key Words: Neology, morphological derivation, type frequency, token frequency, language vitality.

\section{INTRODUCCIÓN}

La creación de palabras nuevas utilizando los recursos propios de la lengua es un indicador de la vitalidad interna de la misma. Al llevar a cabo este proceso, el hablante recurre a reglas gramaticales diversas que tiene incorporadas y las aplica a bases léxicas preexistentes. Si, además, sus creaciones neológicas ${ }^{2}$ reflejan algún tipo de trasgresión de la regla, es decir, algún tipo de 'irregularidad' respecto de la norma, esto demostraría un grado aún mayor de vitalidad lingüística. Como señalan Cabré, Bayà, Bernal, Freixá, Solé y Vallès (2002a:161): “los neologismos no se construyen a través de recursos específicos, sino que utilizan los mismos patrones y recursos que describen las palabras consolidadas en la lengua".

El objetivo de este trabajo fue analizar las propiedades morfológicas y semánticas del sufijo -ero dentro de las creaciones neológicas por sufijación en las variedades del español de Argentina, Chile y Uruguay, como una manera de verificar la vitalidad y productividad de estas variedades. Con la misma finalidad se comparó el significado en aquellos casos en que los sufijos -ero e -ista se adjuntaban a una misma base léxica.

La razón por la que se decidió analizar la productividad del sufijo -ero fue su frecuente utilización en el ámbito deportivo en Chile, en que el sufijo se adjunta a bases existentes alterando las reglas de significado de este. Así, por ejemplo, para denominar un determinado equipo de fútbol, en lugar de utilizar el nombre del mismo, se recurre al procedimiento retórico de meto- 
nimia y se lo denomina utilizando una característica propia de la zona de origen del equipo. Estas denominaciones son sistemáticamente utilizadas por periodistas deportivos tanto de prensa escrita como de radio y televisión, así como también por el público general. Cabe señalar que durante el transcurso de la investigación se decidió ampliar el estudio para determinar en qué otras áreas era recurrente este sufijo.

\section{Marco teórico}

La formación de palabras estudia el análisis y comprensión de los mecanismos por medio de los cuales se crea y se renueva el léxico. Estos mecanismos son principalmente morfológicos, y están referidos a las distintas formas de combinación de palabras, si bien la creación de estas puede incluir otros procedimientos como préstamos de otras lenguas, formación de nuevos términos, es decir, distintos procesos neológicos (Lang, 1992).

Como es sabido, en todas las lenguas se dan estos procesos neológicos que forman parte de la competencia de los hablantes. La descripción de las creaciones neológicas permite observar que los neologismos no se construyen a través de procesos y recursos específicos, sino que utilizan los mismos patrones y recursos que describen las palabras consolidadas en la lengua. Las tres vías esenciales de integración de nuevas unidades en el léxico de una lengua son la creación ex nihilo, la creación formal y semántica, y el préstamo. Entre los mecanismos de formación se dan procesos morfológicos, sintácticos, fonológicos y semánticos, y dentro de los procesos morfológicos, a los que se hará referencia en este artículo, están la derivación por prefijación, la derivación por sufijación y la composición (Cabré et al., 2002a).

Desde la perspectiva de la lingüística diacrónica, Vallès (2002:150) observa:

"un neologismo es una palabra nueva en relación a la lengua empleada por una comunidad lingüística en una época anterior: tiene a la vez, una dimensión social y temporal. Por tanto, para distinguir los neologismos actuales de las palabras ya consolidadas en la lengua, cabe comparar el léxico actual con el empleado anteriormente y considerar neológicas aquellas palabras complejas que solo aparezcan en el corpus más reciente”.

Por su parte, desde el punto de vista del sentido, Alcoba (2007:24) entiende el neologismo como:

"una palabra, una acepción o una frase nueva en el conjunto de unidades comunicativas de que dispone el hablante. 0 sea que se considera neologismo una palabra enteramente nueva, una palabra parcialmente nueva o un giro o combinación de palabras usado con un sentido particular. Lo esencial en el neologismo es el nuevo significado que por distintas necesidades comunicativas del hablante se incorpora al repertorio de palabras 
disponibles con una forma totalmente nueva, con un sentido nuevo en una forma antigua que ya se usaba con otros significados o con una combinación de formas que manifiestan así su nuevo significado".

Varela (2005), al igual que Alcoba (2007), aborda la formación de palabras desde el punto de vista del sentido y distingue entre los recursos utilizados para la creación de palabras nuevas aquellos que se basan en la semejanza por asociación de sentidos o metáfora y los que se basan en la metonimia o asociación entre nombres de objetos que tienen alguna relación de proximidad. Este último recurso dio origen a este trabajo.

Ahora bien, definida la unidad neológica, se aborda a continuación la morfología derivativa como procedimiento para la formación de palabras, específicamente, la derivación mediante afijos, mecanismo utilizado por las lenguas para ampliar el vocabulario.

En palabras de Regúnaga (2005:251) "toda lengua tiene en su haber un conjunto de elementos que constituyen la base de su estructura léxica y sobre ella operan diversos afijos de derivación, que por sufijación, prefijación o infijación generan nuevos elementos léxicos”.

Campanaga (2008), al igual que Cabré et al. (2002a), sostienen, respecto de la capacidad creativa de la lengua española, que una de las formas de mayor productividad de la lengua es la formación de neologismos por derivación y por composición. De ellos, uno de los procedimientos de mayor productividad y vitalidad es la sufijación.

Por lo tanto, la derivación léxica mediante sufijación es el procedimiento de formación de palabras más productivo y variado de nuestra lengua. El español dispone de un número considerable de sufijos, con significados muy variados, y además permite este tipo de derivación en todas las clases de palabras principales; también "emplea la sufijación en todo tipo de lenguaje -técnico y científico, jurídico y administrativo, literario- en todos los registros idiomáticos y tanto en la modalidad oral como en la escrita" (Varela, 2005:41).

Los sufijos tienen, por lo general, una categoría gramatical propia e incluso, en el caso de algunos sufijos nominales, un género fijo; consecuentemente, cuando uno de estos sufijos se adjunta a una base léxica que no coincide con su categoría, cambia la categoría de esta. A su vez, Varela (2005:44) añade:

“un mismo sufijo puede establecer diferentes relaciones semánticas con su base respectiva y tener, por tanto, distintas funciones. Se habla entonces de sufijos 'polifuncionales'; contrariamente, una misma función semántica puede materializarse en diferentes sufijos, a los que consideraremos, por tanto, 'sinónimos'”. 
Para cumplir con el objetivo de este trabajo, se han estudiado las creaciones neológicas que incluyen este sufijo dada su conocida productividad en el español, lo que permite comprobar la frecuencia de las formas (type frequency) o número de palabras distintas que lo contienen, la productividad del mismo o facilidad de activación que tiene un determinado afijo para ser usado en la creación o comprensión de nuevas unidades léxicas o la frecuencia de uso de las palabras que contienen el afijo (token frequency.)

Ahora bien, los medios de comunicación y, por ende, la prensa escrita son los principales propagadores de neologismos, pues reflejan los cambios lingüísticos que se producen en la lengua. “Es, por tanto, la fuente renovadora de léxico más eficaz con la que cuenta el hablante de español para constatar la vitalidad de nuestro idioma” (Ortega Martín, 2001:1). La prensa recoge tanto la neología espontánea y la planificada como la neología general y la especializada. En cuanto a la neología espontánea, Cabré et al. (2002a:164) observan:

"el grado de vitalidad interna de una lengua se puede medir a través del análisis de la frecuencia de uso de los distintos procesos y recursos de creación, considerando que los diversos procesos neológicos se organizan en un eje que representa grados de vigor distintos de un sistema (eje de vitalidad). El polo de máxima vitalidad interna lo ocupan aquellos procesos genuinos del sistema que utilizan sus propios recursos para la creación de nuevas unidades: el locutor utiliza reglas diversas pues considera que estas pertenecen a su propia lengua y, en consecuencia, el receptor decodifica dichas unidades más o menos fácilmente según el grado de productividad de la regla utilizada y la transparencia semántica de la unidad".

Al estudiar el comportamiento del sufijo -ero se debe aplicar la regla de derivación, para lo cual se necesita una base léxica preexistente a la que se aplique la regla. En este caso, Cabré, Freixa y Solé (2002b:132-133) hablan de:

“littéralité d'une règle (seulement règles de dérivation) comme l'application stricte de toutes les conditions de combinaison que cette règle impose à la base lexicale à laquelle un affixe est combiné. Cette littéralité implique une prévisibilité absolue de l'unité lexicale résultante".

Sin embargo, cuando un locutor transgrede las reglas de derivación, esta transgresión no implica la aparición de una regla nueva en la gramática, sino una modificación de las características de una regla existente. Se produce una transgresión, por ejemplo, cuando ocurre una ruptura ante una previsibilidad, lo que hace que el locutor perciba un elemento extraño, raro, distinto del uso acostumbrado. 


\subsection{Valores del sufijo -ero}

A continuación se presentan los distintos valores o significados del sufijo -ero que aparecen en diversas fuentes bibliográficas, para la formación de distintas categorías de palabras.

Bosque y Demonte (1999) en su 'Gramática descriptiva de la lengua española' establecen, en términos generales, que el sufijo -ero es sufijo nominalizador y sufijo que se adjunta a siglas, además de formador de adjetivos denominales y de numerales ordinales.

Con respecto a su función como sufijo nominalizador, los valores en que coinciden todas las fuentes consultadas (Lagore, 1988; Lang, 1992; Santana, Carreras, Pérez \& Rodríguez, 2003; Varela, 2005; Diccionario de la Lengua Española, 2001; Diccionario de uso del español de América y de España, 2002) son los referidos a oficio, ocupación, profesión o cargo; lugar donde abunda, se deposita o guarda algo, y denominación de árbol o planta. Además, Lang (1992) recoge la referencia a un receptáculo, material u objeto y el uso agentivo. Varela (2005), a su vez, también consigna su sentido de recipiente e instrumento y considera además el uso de este sufijo para indicar cualidad o conducta. Por su parte, Lagore (2008) y Santana et al. (2003) agregan el uso como formador de gentilicios.

En lo que respecta a su utilización como formador de adjetivos, las fuentes consignan los sentidos más generales de relación o cualidades y propiedades de personas, animales o cosas, que es muy aficionado a, y que a veces puede tener un matiz despectivo.

Además de los valores de -ero, se estudió también el binomio -ero / -ista en algunas formaciones neológicas encontradas en el corpus para comparar los significados de ambas unidades. Con la finalidad de realizar esta comparación, se contrastaron unidades neológicas con unidades no neológicas en que alternaran dichas formaciones.

Los valores del sufijo -ista repertoriados son los siguientes:

El Diccionario de la Lengua Española (2001) indica los siguientes valores para el sufijo -ista: se usa en adjetivos que habitualmente se sustantivan y suelen significar partidario de o inclinado a lo que expresa la misma raíz con el sufijo -ismo. También forma sustantivos que designan generalmente a la persona que tiene determinada ocupación, profesión u oficio.

El Diccionario de uso del español de América y de España (2002) consigna que el sufijo -ista proviene del griego a través del latín y que sirve para formar sustantivos a partir de sustantivos con el significado de oficio o profesión, partidario de una doctrina, persona que realiza una actividad o que tiene una afición, y persona o cosa que tiene o muestra una actitud.

Lang (1990:180), por su parte, señala que: 
"el campo semántico al que pertenecen las bases a las que se adjunta -ista coincide con el de -ismo e implica habitualmente un paradigma derivativo (Castro $\rightarrow$ castrista $\rightarrow$ castrismo). - Ista es, en principio, agente, y se aplica a miembros representativos de movimientos o a profesiones y, de forma particular, a ocupaciones artísticas y deportistas. Su vitalidad queda reflejada por su tendencia a suplantar lexemas rivales, como por ejemplo finanzas $\rightarrow$ financista = financiero".

En la denominación de profesiones, -ista se opone a -ero, puesto que ambos se adjuntan al mismo tipo de base, indicando el material o el producto que se maneja. La elección entre uno de ellos no está morfológicamente determinada, pero puede depender de preferencias dialectales, por ejemplo, camionero-camionista (Lang, 1990).

\section{Metodología}

El corpus está constituido por las creaciones neológicas que terminan en -ero repertoriadas en prensa escrita de Argentina, Chile y Uruguay, entre los años 2004 y 2006, obtenidas de la base del Observatorio de Neología del IULA de la Universitat Pompeu Fabra de Barcelona (OBNEO), así como de la base de datos de la Antena Chilena, elaborada por el Grupo de Estudios Terminológicos de la Universidad de Concepción, TermUdeC. Los periódicos de los cuales se extrajeron los neologismos fueron La Nación y Clarín de Argentina, El País de Uruguay, y El Mercurio y El Sur de Chile.

En primer lugar, de este corpus se extrajeron todas aquellas unidades terminadas en -ero correspondientes a formación por sufijación y a la categoría de neologismo semántico, excluyéndose todo otro tipo de formación. Además, se seleccionaron las palabras terminadas en el sufijo -ista que eran susceptibles de ser comparadas con sus homólogas terminadas en -ero. En segundo lugar, se realizó una revisión bibliográfica para conocer los distintos valores de estos sufijos registrados en los diversos córpora de exclusión del proyecto Antena Neológica, además de otras fuentes, a saber:

a) Córpora de exclusión del proyecto Antenas Neológicas:

- Real Academia Española. Diccionario de la Lengua Española (2001).

- Diccionario de uso del español de América y España (2002).

- Diccionario de la Lengua Española (2001).

b) Otras fuentes:

- Gramática descriptiva de la lengua española de Bosque y Demonte (1999).

- Formación de palabras en español. Morfología derivativa productiva en el léxico moderno de Lang (1992). 
- Los sufijos -ario, -dor, -ería, -ero, -ista y -orio en los términos artísticos de Lagore (1988).

- Morfología léxica: la formación de palabras de Varela (2005).

- Relaciones morfoléxicas sufijales del español de Santana, Carreras, Pérez y Rodríguez (2003).

- Morfología derivativa: consideraciones en torno al uso de diminutivos en la ciudad de Santa Rosa (La Pampa-Argentina) de Regúnaga (2005).

- Nuevo diccionario ejemplificado de chilenismos y de otros usos diferenciales del español de Chile de Morales Pettorino (2006).

Luego se hizo un análisis exhaustivo de cada una de las unidades repertoriadas con el fin de conocer su significado y observar de qué manera coincidían con los valores registrados en las distintas obras revisadas. Posteriormente, se procedió a agrupar los distintos neologismos de acuerdo a sus significados. Finalmente, se comparó unidades neológicas y no neológicas terminadas en -ero y en -ista adjuntadas a una misma base léxica. En este caso, se presentan solamente ejemplos que dan cuenta de la coocurrencia, se comparan los significados de dichas unidades y, para las unidades neológicas, se consigna el país que las relevó. Las unidades no neológicas se tomaron de las obras lexicográficas que constituyen el corpus de exclusión del proyecto Antenas Neológicas, así como del Nuevo diccionario ejemplificado de chilenismos.

\section{Análisis y discusión de resultados}

Del total de los neologismos encontrados en el corpus, incluidas las repeticiones de cada uno, se recopilaron 147 unidades neológicas procedentes de las fuentes de los tres países, como se puede apreciar en la Tabla 1:

Tabla 1. Cantidad total de ocurrencias neológicas por país terminadas en -ero correspondientes a la formación por sufijación (FSUF) y a neologismos semánticos (S) en valores absolutos y porcentajes.

\begin{tabular}{|c|c|c|c|c|c|c|}
\hline País & \multicolumn{2}{|c|}{$\begin{array}{c}\text { FSUF } \\
\text { casos } \%\end{array}$} & \multicolumn{2}{|c|}{$\begin{array}{c}\mathrm{S} \\
\text { casos }\end{array}$} & \multicolumn{2}{|c|}{$\begin{array}{c}\text { Total } \\
\text { casos \% }\end{array}$} \\
\hline Argentina & 18 & 34,6 & 34 & 65,4 & 52 & 35,4 \\
\hline Chile & 56 & 81,1 & 13 & 18,9 & 59 & 46,9 \\
\hline Uruguay & 15 & 57,7 & 11 & 42,3 & 26 & 17,7 \\
\hline Totales & 89 & 60,5 & 58 & 39,5 & 147 & 100 \\
\hline
\end{tabular}

Al observar los totales, se puede constatar que la mayoría de las unidades corresponden a for- 
mación por sufijación que superan en más de un 20\% las creaciones de neologismos semánticos, es decir, palabras que terminan en el sufijo -ero y que se encuentran consignadas en los córpora de exclusión, pero que han adquirido una nueva acepción.

En la Tabla 2, se puede observar el total de neologismos diferentes sin repetición, correspondiente a las unidades recopiladas en cada uno de los países.

Tabla 2. Cantidad total de ocurrencias neológicas distintas sin repetición por país y tipo de formación en valores absolutos y porcentaje.

\begin{tabular}{|c|cc|cc|cc|}
\hline País & \multicolumn{2}{|c|}{$\begin{array}{c}\text { FSUF } \\
\text { casos } \%\end{array}$} & \multicolumn{2}{|c|}{$\begin{array}{c}\text { S } \\
\text { casos }\end{array}$} & \multicolumn{3}{|c|}{ Total } \\
casos $\%$
\end{tabular}

Estos resultados corroboran una vez más que la formación por sufijación es claramente mayor que la creación de tipo semántico, probablemente porque es más sencillo y expedito para el hablante adjuntar un sufijo ya existente a una base léxica con el propósito de crear una palabra que provea un nuevo significado. Esto vendría a corroborar la idea de que la formación de neologismos por derivación es de alta productividad en la lengua española.

\subsection{Valores del sufijo -ero encontrados en el corpus}

Como ya se ha señalado, las creaciones neológicas con la terminación -ero que se recopilaron del corpus corresponden tanto a formación por sufijación como a neologismos semánticos. Ahora bien, el hecho de que los neologismos semánticos analizados terminen en -ero refuerza la tesis de la alta productividad de este sufijo para formar unidades nuevas, siendo la type frequency la que da cuenta del número significativo de creaciones por derivación mediante este sufijo.

A continuación se presentan los resultados obtenidos con respecto a los neologismos terminados en -ero en los dos tipos de formaciones presentadas anteriormente y según los distintos valores semánticos recogidos, consignándose a partir de la Tabla 3 tanto la type frequency como la token frequency. 
Tabla 3. Valores semánticos de -ero recogidos del corpus y número de ocurrencias, en valores absolutos y porcentaje.

\begin{tabular}{|l|c|c|}
\hline Valores semánticos de -ero & Valor absoluto & Porcentaje \\
\hline Oficio, ocupación o cargo & 66 & $44,9 \%$ \\
\hline Afición a una actividad & 41 & $27,9 \%$ \\
\hline Objeto o función específica & 3 & $2,0 \%$ \\
\hline $\begin{array}{l}\text { Relativo a un equipo de fútbol o de básquetbol } \\
\text { propio de una región productiva }\end{array}$ & 37 & $25,2 \%$ \\
\hline Total & 147 & $100 \%$ \\
\hline
\end{tabular}

De los cuatro valores de -ero repertoriados en el corpus, los tres primeros que se presentan en la Tabla precedente corresponden a las categorías existentes y definidas en las obras gramaticales y lexicográficas consultadas. El desglose de estos valores se puede apreciar en las tablas 4, 5 y 6 que se presentan a continuación. El cuarto valor semántico, sin embargo, transgrede la norma, como se analizará más adelante.

Tabla 4. Valor de -ero que se adjunta a un sustantivo con el significado de persona que tiene un oficio 0 profesión, según tipo de formación, país de origen y frecuencias.

\begin{tabular}{|l|c|c|c|}
\hline \multicolumn{1}{|c|}{ Unidad neológica } & Tipo de formación & País & Frecuencias \\
\hline banderero & FSUF & Chile & 1 \\
banderillero & S & Argentina & 1 \\
cablero & S & Uruguay & 1 \\
cartonero & S & Argentina & 6 \\
cocacolero & FSUF & Argentina & 1 \\
estacionero & Argentina & 4 \\
fletero & S & Argentina & 1 \\
marketero & FSUF & Chile & 1 \\
microbusero & FSUF & Chile & 3 \\
notero & FSUF & Arg. /Chile/Uruguay & $1-4-1$ \\
palenquero & USUF & Uruguay & 2 \\
petisero & FSUF & Chile & 1 \\
pirquinero & S & Uruguay & 2 \\
omnibusero & FSUF & Argentina & 1 \\
portero & FSUF & Argentina & 1 \\
remisero & FSUF & Chile & 4 \\
rondero & FSUF & Argentina & 1 \\
sojero & FSUF & Argentina & 2 \\
tachero & S & Uruguay & 1 \\
tealero & FSUF & Argentina/Uruguay & 1 \\
clonero & FSUF & Uruguay & $20-3$ \\
piquetero & S & & 1 \\
rapiñero & FSUF & Total token frequency: 66 \\
\hline Total type frequency: 23 & \multicolumn{2}{c}{} \\
\hline
\end{tabular}


Nuevamente, la mayoría de las unidades neológicas corresponden a formación por sufijación. Argentina tiene la más alta productividad en este valor de -ero, con 13 de las 23 type frequencies. Pocas unidades se repiten en los tres países de origen de los neologismos, situación que habla de las diferencias culturales entre estas naciones no obstante la cercanía geográfica que presentan. Estas diferencias culturales obedecen a las distintas realidades del desarrollo social y económico de cada país. Con el significado de 'persona que tiene un oficio o profesión' se encuentran bases con distintos significados, que designan lo producido por el que realiza la actividad (sojero, porotero, tealero, clonero, piquetero), el instrumento con el que la realiza (omnibusero, remisero, microbusero, motoquero) o el lugar en el que la desarrolla (estacionero).

Los ejemplos que ilustran los distintos valores de -ero que se presentan a continuación y más adelante en el texto, han sido extraídos de la Base de Datos del Observatorio de Neología (BOBNEO) de Barcelona3:

microbusero adj., FSUF

Un 30 por ciento de evasión se registra en la locomoción colectiva de Valparaíso y Concepción, según estadísticas de los empresarios *microbuseros* de ambas ciudades. X1, 21/09/2008

omnibusero m., FSUF

Los taximetristas y *omnibuseros* realizaron ayer un paro que dejó a mucha gente a pie en el transcurso de la tarde. U2, 22/01/2004 
Tabla 5. Valor de -ero que se adjunta a un sustantivo y que tiene el significado de persona que tiene una afición, según tipo de formación, país de origen y frecuencia.

\begin{tabular}{|l|c|c|c|}
\hline \multicolumn{1}{|c|}{ Unidades } & Tipo de formación & País & Frecuencias \\
\hline bloggero & FSUF & Chile & 1 \\
grafitero & FSUF & Chile & 3 \\
jeepero & FSUF & Chile & 1 \\
motoquero & FSUF & Argentina & 1 \\
puzzlero & Chile & 1 \\
tarrero & FSUF & Chile & 3 \\
arcillero & FSUF & Chile & 1 \\
cestero & FSUF & Chile & 1 \\
polero & S & Argentina & 3 \\
rebotero & FSUF & Chile & 1 \\
skeetero & FSUF & Chile & 2 \\
batuquero & FSUF & Chile & 1 \\
candombero & FSUF & Arg/Chile/Urug & 1 \\
hiphopero & FSUF & Argentina & 1 \\
jazzero & FSUF & Argentina & 1 \\
murguero & FSUF & Uruguay & 1 \\
violinero & FSUF & Uruguay & 1 \\
zanquero & FSUF & Chile & 4 \\
petitero & FSUF & Chile & 4 \\
sesentero & FSUF & Chile & 3 \\
ochentero & FSUF & Chile & 1 \\
ondero & FSUF & \multicolumn{2}{|c|}{ Total token frequency: 41} \\
jetsetero & FSUF & \multicolumn{2}{|c}{}
\end{tabular}

El significado de -ero en este caso se refiere a una persona que practica o es aficionada a un deporte, a un estilo musical, que toca un instrumento, que participa en actividades artísticas, o que tiene afición por tendencias relacionadas con costumbres, modas o épocas. Como se observa en la Tabla 5, casi la totalidad de las creaciones neológicas correspondientes a este valor obedecen a la formación por sufijación, prácticamente ninguna se repite de un país a otro y el país más productivo en este tipo es Chile, con 15 de 23 type frequencies, como se aprecia en los siguientes ejemplos:

\section{candombero $m$. , FSUF}

En este rincón: Rubén Rada y todo su genio *candombero*; en este otro, el bajista Javier Malosetti y su trío, con sus depurados aportes de jazz fusión. N, N, A1, 21/01/2006, ESPEC, 7, 03/05/2007, jaz, 03/05/2007, jaz

petitero m., “ “, FSUF 
Pero lo último que supe de los snobs fue que se llamaban *petiteros*, porque se reunían en el Petit Café de Buenos Aires y usaban mocasines, el pelo largo y los sacos cortos y entallados. 9A, derivado del adjetivo francés "petit", U2, 11/04/2004, CONTR, 28, 28/12/2004, mbr, $30 / 01 / 2006$, obn, 30/01/2006, obn

Tabla 6. Valor de -ero que se adjunta a un sustantivo con el significado de instrumento/objeto que tiene una determinada función, de acuerdo al tipo de formación, país y frecuencia.

\begin{tabular}{|l|c|c|c|}
\hline \multicolumn{1}{|c|}{ Unidades } & Tipo de formación & País & Frecuencias \\
\hline cosmetiquero & FSUF & Chile & 1 \\
jardinero & S & Argentina & 1 \\
parrillero & FSUF & Uruguay & 1 \\
\hline Total type frequency: 3 & \multicolumn{2}{|c|}{ Total token frequency: 3} \\
\hline
\end{tabular}

Este valor de -ero tiene baja productividad y, como en los casos anteriores, predomina la formación por sufijación.

Ahora bien, el último valor de -ero estudiado corresponde a creaciones neológicas cuyo significado escapa a la norma, es decir, que refleja una transgresión a la regla, la que se manifiesta en alguna forma de irregularidad que el hablante puede percibir.

Así, una especificación de la regla es que se toma como base nombres que se emplean para designar un equipo de fútbol o básquetbol y que aluden a una actividad económica característica de la zona de origen del equipo. Esta forma metonímica fue observada por la Antena de Chile y de Uruguay solamente. Así, por ejemplo, 'cafetero' se refiere a la selección de fútbol de Colombia, país productor de café; 'cervecero', al equipo de la ciudad de Quilmes, de donde es originaria la cerveza del mismo nombre; 'ruletero', al equipo de fútbol Everton de Viña del Mar, ciudad conocida por su casino de juegos; 'papayero', al equipo de fútbol de La Serena, ciudad que se vincula al cultivo de las papayas; 'salmonero', al equipo de fútbol de Puerto Montt, en cuya zona existen criaderos de salmón; 'acerero', al equipo de Huachipato de la Compañía de Aceros del Pacífico y 'minero', al equipo de fútbol Cobreloa de Calama, ciudad próxima a la mina de cobre de Chuquicamata. Además, otra restricción tiene que ver con la flexión de las creaciones neológicas, puesto que en general se usan en plural, como se puede observar en los ejemplos presentados más adelante. 
Tabla 7. Valor de -ero que se adjunta a un sustantivo que designa un equipo de fútbol o básquetbol y que alude a una actividad económica característica de la zona de origen del equipo, según tipo de formación, país y frecuencias.

\begin{tabular}{|l|c|c|c|}
\hline \multicolumn{1}{|c|}{ Unidades } & Tipo de formación & País & Frecuencias \\
\hline acerero & FSUF & Chile & 12 \\
aguatero & S & Uruguay & 1 \\
cafetero & S & Chile/Uruguay & $1-1$ \\
carbonero & S & Uruguay & 5 \\
cervecero & S & Chile & 2 \\
darsenero & FSUF & Uruguay & 5 \\
minero & S & Chile & 2 \\
papayero & S & Chile & 2 \\
ruletero & S & Chile & 3 \\
salmonero & FSUF & Chile & 3 \\
\hline \multicolumn{2}{|r|}{ Total type frequency: 10} & \multicolumn{2}{|c|}{ Total token frequency: 37 } \\
\hline
\end{tabular}

Ejemplos:

acerero $m$., FSUF

El elenco de la usina desperdició una gran oportunidad para comenzar a abrochar la clasificación a la Copa Sudamericana. Ante un equipo que jugó 74 minutos con un hombre menos, los *acereros* igualaron a dos.

9Rd, relativo al equipo de fútbol Huachipato de la Compañía de Aceros del Pacífico, X1, 07/05/2006, DEP, 4, 19/12/2006, mfm, 28/02/2007, mfm, 22/03/2007, acp

\section{cervecero $\mathrm{m}$., $\mathrm{S}$}

Pudo liquidarlo Miguel Caneo para los *cerveceros*, pero la "U" sacó toda su garra para irse arriba con escaso fútbol y reclamar los puntos que le robaban desde su casa.

4/9Rd, neologismo semántico respecto de la acepción 3 (DRAE); relativo al club de fútbol Quilmes, X2, 23/02/2005, DEP, 13, 01/06/2005, cge, 02/06/2006, mba, 02/06/2006, mba

En las creaciones neológicas incluidas en la Tabla 4 se puede constatar que se ha producido una ruptura de la previsibilidad, ya sea porque algunas unidades han adquirido nuevos valores, o se ha producido un cambio o una ampliación de sentido, ya sea porque las unidades nuevas se perciben como distintas, raras o extrañas por parte del receptor, aun cuando el proceso de formación de palabras se apegue a la norma. 


\subsection{Comparación de las unidades neológicas terminadas en -ero e -ista}

Analizados los distintos valores del sufijo -ero, pareció interesante comparar las unidades léxicas terminadas en -ero e -ista adjuntas a una misma base. En estos pares de palabras las unidades son una de ellas neológica y la otra no neológica.

3.2.1. Comparación de unidades neológicas terminadas en -ista con unidades no neológicas terminadas en -ero

La comparación que se realiza a continuación corresponde a los neologismos terminados en -ista con unidades no neológicas terminadas en -ero. Se hace la salvedad de que en este caso se comparan solo algunos ejemplos interesantes y no todas las unidades repertoriadas. Como se puede observar en la Tabla 8, para unidades como 'bombista', 'platista', 'santista' existe una unidad terminada en -ero con la cual no tienen nada en común.

Tabla 8. Comparación del significado de unidades neológicas terminadas en -ista con unidades no neológicas terminadas en -ero.

\begin{tabular}{|l|l|c|}
\hline \multicolumn{1}{|c|}{ Unidades } & \multicolumn{1}{|c|}{ Significado } & País \\
\hline $\begin{array}{l}\text { platista/ } \\
\text { platero }\end{array}$ & $\begin{array}{l}\text { platista: que se mueve por el dinero. } \\
\text { platero: persona que trabaja el metal, plata, que vende objetos labrados de } \\
\text { plata u oro, o joyas con pedrería. }\end{array}$ & Chile \\
\hline $\begin{array}{l}\text { bombista/ } \\
\text { bombero }\end{array}$ & $\begin{array}{l}\text { bombista: que toca el bombo. } \\
\text { bombero: operario encargado de extinguir los incendios, persona que tiene } \\
\text { por oficio trabajar con la bomba hidráulica. }\end{array}$ & Argentina \\
\hline $\begin{array}{l}\text { violista / } \\
\text { violero }\end{array}$ & $\begin{array}{l}\text { violista: que toca la viola. } \\
\text { violero: mosquito, insecto díptero. }\end{array}$ & Chile \\
\hline $\begin{array}{l}\text { charanguista/ } \\
\text { charanguero }\end{array}$ & $\begin{array}{l}\text { charanguista: que toca el charango. } \\
\text { charanguero: chapucero, es decir, hecho tosca y groseramente. }\end{array}$ & Argentina \\
\hline $\begin{array}{l}\text { santista/ } \\
\text { santero }\end{array}$ & $\begin{array}{l}\text { santista: relativo al equipo de fútbol Santos. } \\
\text { santero: dicho de una persona: que tributa a las imágenes un culto } \\
\text { supersticioso, que cuida de un santuario, que pide limosna, llevando de casa } \\
\text { en casa la imagen de un santo, que pinta, esculpe o vende santos. }\end{array}$ & Argentina \\
\hline $\begin{array}{l}\text { velista/ } \\
\text { velero }\end{array}$ & $\begin{array}{l}\text { velista: deportista de embarcación a vela. } \\
\text { velero: dicho de una persona que asiste a velas y romerías, que hace velas } \\
\text { o las vende. }\end{array}$ & Argentina \\
\hline $\begin{array}{l}\text { vientista/ } \\
\text { ventero }\end{array}$ & $\begin{array}{l}\text { vientista: relativo a los instrumentos de viento. } \\
\text { ventero: persona que tiene a su cuidado y cargo una venta para hospedaje } \\
\text { de los pasajeros. }\end{array}$ & Argentina \\
\hline $\begin{array}{l}\text { balista/ } \\
\text { balero }\end{array}$ & $\begin{array}{l}\text { balista: deportista que lanza la bala. } \\
\text { balero: juguete; también inteligencia, capacidad de entender. }\end{array}$ & Chile \\
\hline
\end{tabular}


Vale la pena destacar que los significados contrastados aquí son completamente distintos uno del otro. Para estas unidades no se registraron casos en el español de Uruguay.

En la Tabla 9 se puede observar los pares -ero/-ista que, si bien no tienen exactamente el mismo significado, guardan una relación estrecha entre sí. Por ejemplo, en el par canoero/ canoísta, el rasgo compartido es la canoa.

Tabla 9. Unidades neológicas terminadas en -ista y unidades no neológicas terminadas en -ero con relación temática.

\begin{tabular}{|l|l|c|}
\hline Unidades & \multicolumn{1}{|c|}{ Significado } & País \\
\hline $\begin{array}{l}\text { canoísta/ } \\
\text { canoero }\end{array}$ & $\begin{array}{l}\text { canoísta: deportista de canoa. } \\
\text { canoero: persona que gobierna la canoa. }\end{array}$ & Chile \\
\hline $\begin{array}{l}\text { vientista/ } \\
\text { ventero }\end{array}$ & $\begin{array}{l}\text { vientista: relativo a los instrumentos de viento. } \\
\text { ventero: adj. que ventea. }\end{array}$ & Argentina \\
\hline $\begin{array}{l}\text { violista / } \\
\text { violero }\end{array}$ & $\begin{array}{l}\text { violista: que toca la viola. } \\
\text { violero: constructor de instrumentos de cuerda. }\end{array}$ & Argentina \\
\hline $\begin{array}{l}\text { mercadista/ } \\
\text { mercadero }\end{array}$ & $\begin{array}{l}\text { mercadista: relativo al mercado. } \\
\text { mercadero: en desuso, mercader. }\end{array}$ & Argentina \\
\hline $\begin{array}{l}\text { velista/ } \\
\text { velero }\end{array}$ & $\begin{array}{l}\text { velista: deportista de embarcación a vela. } \\
\text { velero: dicho de una embarcación muy ligera o que } \\
\text { navega mucho; fabricante de velas para buques. }\end{array}$ & Chile \\
\hline
\end{tabular}

Si bien estas unidades derivan de una misma base léxica, no tienen exactamente el mismo significado. Nuevamente se constata que de estas unidades no se registraron casos en el español de Uruguay.

Por otra parte, se encontró solo una unidad neológica terminada en -ista que tenía un homólogo no neológico terminado en -ero, en cuyo caso no se presentó una variación de significado, sino que solo se trató de una variante estilística. Esta unidad corresponde al binomio violista/ violero detectado por Chile, en que violista significa 'que toca la viola' y violero, ‘músico que toca la viola o vihuela'. 
3.2.2. Comparación de unidades neológicas terminadas en -ero con unidades no neológicas terminadas en -ista

A continuación se muestran neologismos terminados en -ero y sus respectivos homólogos no neológicos terminados en -ista.

Tabla 10. Neologismos terminados en -ero y unidades no neológicas terminadas en -ista con relación temática pero distinto significado.

\begin{tabular}{|l|l|c|}
\hline \multicolumn{1}{|c|}{ Unidades } & \multicolumn{1}{|c|}{ Significado } & País \\
\hline $\begin{array}{l}\text { jazzero/ } \\
\text { jazzista }\end{array}$ & $\begin{array}{l}\text { jazzero: relativo al jazz. } \\
\text { jazzista: intérprete de jazz. }\end{array}$ & Chile \\
\hline $\begin{array}{l}\text { violinero/ } \\
\text { violinista }\end{array}$ & $\begin{array}{l}\text { violinero: violinista que toca música folclórica. } \\
\text { violinista: persona que toca el violín. }\end{array}$ & Argentina \\
\hline cablero/ & $\begin{array}{l}\text { cablero: persona que instala conexiones a plataformas inalámbricas para } \\
\text { cables del interior (wireless, Internet). } \\
\text { cablista: persona que se dedica a instalar cables eléctricos o de } \\
\text { comunicación. }\end{array}$ & Uruguay \\
\hline
\end{tabular}

Como se puede observar en la Tabla 10, si bien las unidades no tienen el mismo significado, guardan una estrecha relación entre sí. Por ejemplo, en el par jazzista/jazzero, el rasgo semántico compartido es la base léxica 'jazz'.

Por otra parte, se comparó algunas unidades neológicas terminadas en -ero con unidades no neológicas terminadas en -ista, que tenían el mismo significado. Fue el caso de batero/baterista, en que batero es una variante morfológica de baterista y baterista es 'el músico que toca la batería', y las unidades polero/polista que portan el significado de 'jugador de polo'. En estos dos casos los hablantes utilizan indistintamente tanto la unidad neológica como la no neológica, lo que demuestra la vitalidad de ambos sufijos. La elección de uno u otro no está determinada por su morfología, sino más bien parece depender de preferencias geolectales.

Finalmente, se encontró solo una unidad neológica terminada en -ero que tenía un homólogo no neológico terminado en -ista sin relación temática y con distinto significado. Esta unidad corresponde al par barrero/barrista detectado por Chile, en que barrero significa 'persona que apoya a un equipo de fútbol' y barrista, 'artista de circo que trabaja en las barras fijas'. 


\section{CONCLUSIONES}

En primer lugar, respecto de la token frequency en el proceso de sufijación estudiado, se ha podido constatar que muchos neologismos tienen una frecuencia de aparición 1 en el corpus; sin embargo, los usuarios de la lengua tienen conciencia de que se trata de unidades que no son desconocidas, sino que ampliamente utilizadas. La baja ocurrencia, como señala Vallès (2002: 147) "es un indicio de la frecuencia de activación de los afijos".

En segundo lugar, es posible concluir que en algunos neologismos terminados en -ero se ha producido una ruptura de la previsibilidad debido, fundamentalmente, a que algunas unidades han adquirido nuevos valores, a que se ha producido un cambio o una ampliación de sentido, o a que las unidades nuevas se perciben como distintas, raras o extrañas por parte del hablante a raíz de alguna transgresión de las reglas.

Es así como el hallazgo de un valor semántico de -ero que transgrede la norma, es decir, que no se ajusta a los valores semánticos repertoriados en las obras consultadas, permite concluir que el hablante se arroga la licencia de asignar un nuevo valor a un sufijo y crear palabras cuyo fin es denominar realidades nuevas o estilos nuevos de referirse a realidades conocidas. Es el caso específico de las formas metonímicas formadas con una base más el sufijo -ero para designar equipos deportivos en función del lugar de origen, el cual es identificado con alguna área de la producción que caracteriza a la zona.

Desde el punto de vista semántico, se constató que el valor de -ero que corresponde a oficio o profesión es el de más alta productividad, cuestión que se puede atribuir al surgimiento de nuevas actividades en distintos ámbitos que es preciso denominar. Adicionalmente, se puede concluir que las nuevas creaciones formadas con -ero corresponden tanto a la categoría de neología denominativa como a la categoría de índole estilística.

Por otra parte, desde la perspectiva del tipo de formación, las nuevas creaciones que incluyen la terminación -ero corresponden tanto a neologismos semánticos como a neologismos por sufijación, siendo el nivel de productividad más alto en estos últimos. Se puede observar que la mayoría de las unidades son neologismos formados por reglas ya productivas en la lengua; en cambio, en el caso de los neologismos que motivaron este estudio, se trata de unidades que son el resultado de alguna modificación o especificación de reglas ya existentes.

El hecho de que la terminación -ero sea muy productiva se debe a que pareciera estar integrada a la gramática de los hablantes, razón por la que estos recurren a ella con mucha facilidad. A la vez, -ero parece no ser excluyente, sino que dicha forma puede convivir con otra denominación de una misma realidad, como -ista por ejemplo, sin que una forma anule a la otra. 
En cuanto a la comparación de las formas terminadas en -ero e -ista con la misma base léxica, se puede concluir que la creación de tales unidades obedece a las siguientes razones: a) la necesidad de denominación de nuevas realidades, b) la necesidad de darle un nuevo sentido o extensión del significado a las unidades ya existentes y c) una simple variante morfológica que puede obedecer a preferencias geolectales.

Respecto de la vitalidad del español asociada a la modificación de la literalidad de las reglas morfológicas, se evidencia que la productividad de un recurso atañe no solo a su uso sino también a la pluralidad de valores que puede transmitir. En efecto, la especificación de las reglas de sufijación con -ero manifiesta alta polisemia, lo que permite sostener que el aumento de esta en los afijos es un elemento indicador de la vitalidad lingüística interna.

Desde el punto de vista de la productividad de las unidades neológicas terminadas en -ero, ya sea por sufijación o por creación semántica, se pudo constatar que de los tres países, Chile es el que relevó más unidades, seguido de Argentina y luego de Uruguay. Con este hecho no se puede afirmar que la vitalidad de -ero sea mayor en un país que en otro, sino solo que el proceso de pesquisa ha estado orientado a distintas unidades en cada una de las Antenas y de este modo la productividad pareciera ser más alta en Chile. Sin embargo, se comprobó que las creaciones terminadas en -ero son vitales en las tres variedades.

Finalmente, la intención de este estudio es contribuir al análisis de los procesos de formación de neologismos desde el punto de vista tanto morfológico como semántico y, de esta manera, aportar a uno de los objetivos principales del Proyecto Antenas Neológicas que es el enriquecimiento y la actualización de las obras lexicográficas existentes mediante la detección de unidades neológicas que designan nuevas realidades como también de nuevas acepciones atribuidas a unidades ya existentes en la lengua española.

\section{REFERENCIAS BIBLIOGRÁFICAS}

Alcoba, S. (2007). Autorización y uso del neologismo. En R. Sarmiento \& F. Vilches (Eds.), Neologismos y sociedad del conocimiento (pp.23-47). Barcelona: Ariel.

Battaner, P. (2002). Diccionario de uso del español de América y de España. VOXUSO. Barcelona: Spes Editorial.

Battaner, P. (2001). Diccionario de la Lengua Española. LEMA. Barcelona: Spes Editorial, S. L.

Bosque, I. \& Demonte, V. (1999). Gramática descriptiva de la lengua española. Madrid: Espasa Calpe.

Cabré, M., Bayà, R., Bernal, E., Freixa J., Solé, E. \& Vallès, T. (2002a). Evaluación de la vitali- 
dad de una lengua a través de la neología: A propósito de la neología espontánea y de la neología planificada. En M. Cabré, J. Freixa \& E. Solé. (Eds.), Lexic i Neologia. Lexic i Neologia (pp.159-201). Barcelona: Observatori de Neologia. Institut Universitari de Lingüística Aplicada, Universitat Pompeu Fabra.

Cabré, M., Freixa J. \& Solé, E. (2002b). À la limite des mots construits possibles. En M. Cabré, J. Freixa \& E. Solé. (Eds.), Lexic i Neologia (pp.121-138). Barcelona: Observatori de Neologia. Institut Universitari de Lingüística Aplicada, Universitat Pompeu Fabra.

Campanaga, P. (2008). Productividad y registro de las formas derivativas y compositivas. Università di Bologna [en línea]. Disponible en: http://cvc.cervantes.es/literatura/aispi/ pdf/14/14 101.pdf

Lagore, F. (1988). Los sufijos -ario, -dor, -ería, -ero, -ista y -orio en los términos artísticos. Alazet: Revista de Filología. Editores. Instituto de Estudios Altoaragoneses [en línea]. Disponible en: http://www.iea.es/

Lang, M. (1992). Formación de palabras en español. Morfología derivativa productiva en el léxico moderno. Madrid: Cátedra.

Morales Pettorino, F. (2006). Nuevo diccionario ejemplificado de chilenismos y de otros usos diferenciales del español de Chile. Valparaíso: Editorial Puntángeles.

OBNEO. (2008). Observatori de Neología del Institut Universitari de Lingüística Aplicada de la Universitat Pompeu Fabra, Barcelona [en línea]. Disponible en: http://obneo.iula.upf. edu/pobneo/ficha_lista.php

Ortega Martín, M. (2001). Neología y prensa: Un binomio eficaz. Espéculo: Revista de Estudios Literarios [en línea]. Disponible en: http://www.ucm.es/info/especulo/numero18/ neologism.html

Real Academia Española (2001). Diccionario de la Lengua Española. Vigésimo Segunda Edición. Madrid: Espasa Calpe.

Regúnaga, A. (2005). Morfología derivativa: Consideraciones en torno al uso de diminutivos en la ciudad de Santa Rosa (La Pampa-Argentina). Anclajes [en línea]. Disponible en: http://www.biblioteca.unlpam.edu.ar/pubpdf/anclajes/n09a13regunaga.pdf

Santana, O., Carreras F., Pérez. J. \& Rodríguez, R. (2003). Relaciones morfoléxicas sufijales del español. Procesamiento del Lenguaje Natural, 30, 1-73.

Vallès, T. (2002). La productividad morfológica en un modelo dinámico basado en el uso y en los usuarios. En M. Cabré, J. Freixa \& E. Solé. (Eds.), Lexic i Neologia (pp.139-157). Barcelona: Observatori de Neologia. Institut Universitari de Lingüística Aplicada, Universitat Pompeu Fabra.

Varela, S. (2005). Morfología léxica: La formación de palabra. Madrid: Gredos. 


\section{NOTAS}

1 Este artículo es parte de un trabajo mayor denominado 'Productividad de los recursos de neología formal en el español del Cono Sur', presentado en el Congreso Internacional de Neología en Lenguas Románicas (CINEO), Institut Universitari de Lingüística Aplicada de la Universitat Pompeu Fabra de Barcelona, 7 al 10 de mayo de 2008. En dicha investigación participaron Andreína Adelstein, Marina Berri y Andrea Bohrn por la Antena Argentina, CONICET/Universidad Nacional de General Sarmiento; Sara Álvarez por la Atena Uruguaya y la Universidad de la República; además de la Antena Chilena del Grupo de Estudios Terminológicos de la Universidad de Concepción, integrada por las autoras de este artículo.

2 El Proyecto de Antenas Neológicas es dirigido por Judit Freixa de la Universidad Pompeu Fabra (Barcelona) y es financiado por el Observatorio de Neología del Institut Universitari de Lingüística Aplicada de la Universitat Pompeu Fabra, la editorial SPES VOX Bibliograf y Unión Latina. Los nodos que constituyen actualmente este proyecto son el Instituto del Desarrollo Humano de la Universidad Nacional de General Sarmiento (Argentina), la Oficina de Unión Latina (Cuba), el Colegio de México (México), el grupo de terminología de la Facultad de Derecho de la Universidad de la República (Uruguay), el grupo de estudios terminológicos PERUTERM de la Universidad Femenina del Sagrado Corazón (Perú) y el grupo de estudios terminológicos de la Universidad de Concepción TermUdeC (Chile).

3 La Base de Datos del Observatorio de Neología (BOBNEO) utiliza una ficha de vaciado que exige la utilización de una determinada nomenclatura. Como se puede observar en los ejemplos presentados, FSUF significa neologismo formado por sufijación; S neologismo semántico; en el campo de notas, el número 2 indica una variante respecto del neologismo que recoge el diccionario consultado: $2 a$ variante ortográfica, $2 c$ equivalente castellano, etc. La nota 9 incluye subgrupos codificado $9 \mathrm{~A}$ con los comentarios que se estimen pertinentes y $9 \mathrm{R}$ neologismos que pueden parafrasearse 'relativo a'. En cuanto a los diarios chilenos, El Mercurio está codificado como X1 y El Sur, X2; U1 corresponde a El País de Uruguay; A1 a La Nación y A2 al Clarín de Argentina. Las áreas temáticas de los periódicos también están codificadas (OPIN, DEP, ECON, etc.) así como el autor, revisor y validador de cada ficha, por ejemplo: ape, cge, kgo, mfm y pca, para la Antena Chilena. 